\title{
Comparison of Economically Favourable and Further Development Friendly DNA Isolation Methods from Microbial Cultures
}

\author{
Barbara Bánkuti¹, Zoltán Tudós², Susan Szathmary², László Stipkovits³, Zsófia Sipos-Kozma²,

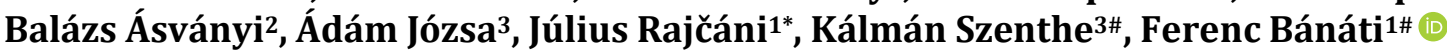

${ }^{1}$ RT-Europe Nonprofit Research Center, Mosonmagyaróvár, Hungary

${ }^{2}$ Galen-Bio Ltd., Mosonmagyaróvár, Hungary

${ }^{3}$ Carlsbad Research Organization, Újrónafö, Hungary

Email: *viruraj@savba.sk

How to cite this paper: Bánkuti, B., Tudós, Z., Szathmary, S., Stipkovits, L., Sipos-Kozma, Z., Ásványi, B., Józsa, Á., Rajčáni, J., Szenthe, K. and Bánáti, F. (2020) Comparison of Economically Favourable and Further Development Friendly DNA Isolation Methods from Microbial Cultures. Advances in Microbiology, 10, 1-13.

https://doi.org/10.4236/aim.2020.101001

Received: December 5, 2019

Accepted: December 31, 2019

Published: January 3, 2020

Copyright ( 2020 by author(s) and Scientific Research Publishing Inc. This work is licensed under the Creative Commons Attribution International License (CC BY 4.0).

http://creativecommons.org/licenses/by/4.0/

\begin{abstract}
Microorganisms, regardless of whether pathogenic or not, may cause enormous economic losses due to adverse effects on human and animal health, or by damaging the quality of the agricultural and food products. Based on these effects, the development of prompt molecular methods and their involvement in the practical pathogen diagnostic diagnostics is more than actual. This paper is focused on the evaluation of easy-to-perform and highly budget-friendly, PCR-related DNA purification protocols for diagnostic purposes especially in water or similar simple matrices. The slight modifications of earlier described DNA isolation methods, which rely on chelate exchange resin and/or ethanol-sodium-based heat lysis, we reevaluated in comparison with a widely used commercial kit. The efficiency of DNA purification techniques was assessed from Gram-negative as well as Gram-positive bacteria and yeast using quantitative PCR. The effectivity of different methods tested may vary depending on the bacterial or yeast species in question. Nevertheless, in our hands, the chelate exchange resin-based methods were found to be the most robust and/or satisfying at least by an acceptable reproducibility rate. Our presented results support the potential of low-cost but still sensitive molecular microbe detection procedures consisting of only a few pipetting steps resulting in good reproducibility and the least possible environmental burden, serving as a good starting point for developments of matrix-specific processes and methods.
\end{abstract}

\section{Keywords}

Microbe Detection, DNA Purification, Lysis, qPCR, Developmental Potential

${ }^{\#}$ Authors contributed equally to the work. 


\section{Highlights}

- The need to simplify the diagnostic methods utilizing DNA isolation along with cost reduction.

- Simple DNA purification methods may serve the matrix specific approaches and automatization.

- Comparative study on DNA purification methods from microbial cells.

- Innovative simplification of diagnostic methods is beneficial regarding time and expenses.

\section{Introduction}

Identification of pathogenic microorganisms using classical cultivation methods is often time-consuming and gives inadequate results that have to be verified via further and sometimes long-lasting cultivation steps [1] [2]. Based on historical reasons and the relatively cheap component prices, this is still at practice in most laboratories, especially those connected to agriculture and animal or human healthcare. In past decades, the development of molecular biological methods and the continuously broadening bioinformatic background promoted the emergence of improved microbe detection methods [1] [3]. The widely available nucleic acid amplification-based detection and identification methods coupled with foregoing cost-effective and rapid DNA isolation protocols allow the completion of the entire diagnostic procedure within a day or even in several hours [4]-[10]. Microbe detection may also rely on microbe-related proteomic characteristics or microbe-specific immune responses. In addition to the widely used and accepted ELISA techniques, many other instrument-dependent solutions are accessible recently [11]. Among others, the wide distribution of mass spectrometry techniques and the continuously broadening, supporting databases supply the opportunity of extremely sensitive and fast microbe detection, although their standardization is quite labor-intensive and often matrix-specific [12] [13] [14]. Among recently published papers, there are several results using Bio-layer interferometry to detect and identify microbes out of different sources but it is facing the same difficulties as mentioned above [15] [16]. The unrelenting need for rapid and reliable methods in the diagnostics of pathogens and indicator microorganisms have always existed, because the quickly achieved results reduce economical losses, shorten the action/reaction time along with protecting animal and human health and the market value of distinct products due to the opportunity for early intervention [17] [18]. Drinking water, being the most important and indispensable product of food industry produced in enormous volumes is of high priority to be free of pathogens.

Regarding sample handling, following or instead of microbe enrichment, one strategy of quick and economical nucleic acid purification is simply to lyse the cells by heating under hypo-osmotic circumstances with simultaneous depletion of PCR inhibitors using Chelate exchange resin, e.g. Chelex-100. This method is quite user-friendly due to the limited number of pipetting steps and, in addition, 
it gives considerably good qPCR results [4]-[9]. Other potential way is to lyse microbes in ethanol containing denaturing alkaline solution (EtNa-Ethanol and $\mathrm{NaOH}$ ) to directly precipitate the deliberated DNA and separate by centrifugation. At the same time, soluble, undesired materials having inhibitory effect on PCR reaction are washed away, providing relatively pure DNA with only several pipetting steps within half an hour [10]. These alternatives are suitable substituents of commercial silica-based methods with comparable results, shorter or equal processing times, less pipetting steps beside their meaningful financial benefit. Here, we report the evaluation of these methods supplemented with slight modifications in microbial DNA purification from physiological salt solution or water inoculated with microbial colony compared to a widely used commercial kit. The osmotic stress caused by pure water facilitates the lysis of microbes and as a consequence, result in even more efficient DNA purification and better DNA yields. The results presented here aim to encourage the use of simple, earlier described methods as a point of origin in diagnostic developments and promote simplification of methods giving satisfying results even in matrix-specific situations. As model organisms for demonstrative purposes, we examined two Gram-positive (Listeria monocytogenes, Staphylococcus aureus) and two Gram-negative (Escherichia coli and Salmonella enterica) pathogenic species which are important in food safety being very common indicator strains, and a yeast $Z$ ygosaccharomyces bailii that can cause food spoilage and thereby economic damages [19]-[24].

\section{Materials and Methods}

\subsection{Microbe Strains and Culturing}

Strains used in the study and the corresponding accession numbers are listed in Table 1. The origin of the strains was the National Collection of Agricultural and Industrial Microorganism (Budapest). The strains in the lyophilized vial were rehydrated for 30 minutes in two $\mathrm{ml}$ rehydration medium according to the manufacturer's recommendation. After rehydration, we prepared pure cultures of given strains by means of spread-plate method [25]. The selective medium for this purpose and the corresponding culturing conditions are listed in Table 2. In each experiment a 24-hour culture was used for the typical biochemical reactions. The final cell concentrations of the suspensions were around $10^{7}-10^{8}$ $\mathrm{ml}^{-1}$.

\subsection{DNA Isolation}

Using alternative methods three $\mathrm{ml}$ saline solution was inoculated with cultured microbes and $100-100 \mu \mathrm{l}$ samples were processed in triplicates, in parallel and repeated twice to collect enough data for the evaluation of effectiveness and reproducibility of the methods.

1) As control method for Gram-positive, Gram-negative bacterial strains and the yeast strain GeneJET Genomic DNA Purification Kit (Thermo scientific, 
Table 1. Microbial strains and the rehydration medium used in the study.

\begin{tabular}{|c|c|c|}
\hline Strain & Medium for rehydration & Manufacturer \\
\hline Escherichia coli (ATCC 11775) & Trypto-Casein Soy Broth (TSB) & Biokar Diagnostics \\
\hline Salmonella enterica (ATCC 13311) & Trypto-Casein Soy Broth (TSB) & Biokar Diagnostics \\
\hline Listeria monocytogenes (ATCC 15313) & Brain Hearth Infusion Broth (BHI) & Biolab \\
\hline Staphylococcus aureus (ATCC 25923) & Trypto-Casein Soy Broth (TSB) & Biokar Diagnostics \\
\hline $\begin{array}{c}\text { Zygosaccharomyces bailii } \\
\text { (ATCC 58445) }\end{array}$ & $6.5 \%$ physiological saline solution & VWR International \\
\hline
\end{tabular}

For comparison of DNA extraction techniques lyophilized gram-negative, gram-positive species and yeast were selected. Medium recommended by the manufacturer was used for recovery.

Table 2. Culturing conditions of the strains used in this study.

\begin{tabular}{cccccc}
\hline & & & \multicolumn{2}{c}{ Incubation } \\
\cline { 3 - 5 } Strain & Selective medium for culturing & Manufacturer & Temperature & Time \\
\cline { 3 - 5 } & & & $\left({ }^{\circ} \mathrm{C}\right)$ & $($ hour) \\
Escherichia coli (ATCC 11775) & Tryptone bile x-glucuronide agar (TBX) & VWR & $44 \pm 1$ & $24 \pm 4$ \\
Salmonella enterica (ATCC 13311) & Xylose lysine deoxycholate agar (XLD) & VWR & $37 \pm 1$ & $24 \pm 4$ \\
Listeria monocytogenes (ATCC 15313) & Agar Listeria acc. to Ottaviani \& Agosti (ALOA) & Biolab & $37 \pm 1$ & $24 \pm 4+24 \pm 4$ \\
Staphylococcus aureus (ATCC 25923) & Baird-Parker agar (BP) & Biolab & $37 \pm 1$ & $24 \pm 4+24 \pm 4$ \\
Zygosaccharomyces bailii (ATCC 58445) & Malt Extract Agar (MEA) & Biolab & $25^{\circ} \mathrm{C}$ & $72-120$ \\
\hline
\end{tabular}

Prior to DNA extraction, the microorganisms were cultured on the selective media under the conditions listed.

\#K0721) was used according to the manufacturer's instruction. The chosen commercial DNA isolation method reflected our previous experience in DNA purification. It worked well, at least in our hands, when compared to another equivalent products coming from different manufacturer, especially for simple food matrices.

2) Chelex-100 based methods rely on the description by Walsh et al. with minor adaptations as shown below [4].

a) Direct Chelex: $100 \mu \mathrm{l}$ 20\% Chelex-100 (Bio-Rad) was added directly to 100 $\mu \mathrm{l}$ sample, vortexed vigorously and incubated for 10 minutes at $100^{\circ} \mathrm{C}$ with shaking at $1000 \mathrm{rpm}$. Samples were centrifuged for one minute at $10.000 \mathrm{~g}$ and the supernatant was used in the subsequent $\mathrm{QPCR}$ reaction.

b) Chelex: $100 \mu \mathrm{l}$ sample was first pelleted at $6.000 \mathrm{~g}$ for six minutes, supernatant was discarded and the pellet was resuspended in $200 \mu \mathrm{l} 10 \%$ Chelex-100. Sample was treated as described above.

3) EtNa-related methods are based on the description of Vingataramin and Frost [10]. The background of this approach is the alkaline $(\mathrm{NaOH})$ lysis of cells and the ethanol-based precipitation of the deliberated DNA molecules in a single step (EtNa: Ethanol-NaOH).

a) Direct EtNa: $455 \mu \mathrm{l} \mathrm{EtNa} \mathrm{solution} \mathrm{was} \mathrm{added} \mathrm{to} 100 \mu \mathrm{l}$ sample, vortexed and incubated at $80^{\circ} \mathrm{C}$ for 10 minutes with continuous shaking with $1000 \mathrm{rpm}$. The 
complete solution was cooled on ice for five minutes and centrifuged at $4{ }^{\circ} \mathrm{C}$ with $16.000 \mathrm{~g}$ for 10 minutes. Pellet was dissolved in EtNa resuspension buffer as described in the original paper.

b) Direct EtNa-Chelex: this procedure was performed as described above (3.a) using a resuspension solution containing $10 \%$ Chelex-100 to deplete the traces of residual PCR inhibitors.

c) Direct EtNa-column: column method was done according to direct EtNa (3.a) except for the cooled solution was loaded onto the column of the GeneJET Genomic DNA Purification Kit. The DNA was eluted (without washing) into $200 \mu$ l elution buffer.

d) EtNa, e) EtNa-Chelex, f) EtNa-column methods were performed as described above (3.a, b, c), but the microbial samples were first pelleted at $6.000 \mathrm{~g}$ for six minutes, the supernatants were discarded and the bacterial pellets were resuspended in $455 \mu \mathrm{l} \mathrm{EtNa}$ solution.

\section{Real-time PCR}

The quantity of DNA obtained by the chosen purification methods were determined by comparing the $\mathrm{Cq}$ values of $\mathrm{qPCR}$ measurements. Other methods reflecting DNA purity were not applied as the Chelex methods, as a consequence of the methodological concept behind, give dirty but well amplifiable samples. The amplifications were performed by real-time PCR using SYBR-Green master mix (Bioline, SensiFAST ${ }^{\mathrm{m} x} \mathrm{SYBR}^{\circledR}$ No-ROX Kit) and genus specific oligos listed in Table 3. The sequences of the listed oligos and their exact design were based on earlier publications [26] [27] [28] [29]. All of the reactions were preceded by a two minutes denaturation step at $95^{\circ} \mathrm{C}$, followed by 40 cycles of amplification, consisting of a 10 seconds denaturation at $95^{\circ} \mathrm{C}$ and a 30 seconds annealing and extension at $60^{\circ} \mathrm{C}$. Melting curve analysis was performed thereafter in order to confirm specific amplification.

Table 3. List of oligos used in qPCR assays in the study.

\begin{tabular}{|c|c|c|c|}
\hline Microbe & $\begin{array}{c}\text { Accession } \\
\text { number }\end{array}$ & $\begin{array}{c}\text { Primer } \\
\text { orientation }\end{array}$ & Primer sequences $\left(5^{\prime}-3^{\prime}\right)$ \\
\hline \multirow{2}{*}{ Escherichia coli } & \multirow{2}{*}{$\begin{array}{l}\text { NCAIM B.01874 } \\
\text { (ATCC 11775) }\end{array}$} & forward & CGACCAAAGCCAGTAAAGTAG \\
\hline & & reverse & AGCCAAAAGCCAGACAGAGT \\
\hline \multirow{2}{*}{ Salmonella enterica } & \multirow{2}{*}{$\begin{array}{l}\text { NCAIM B.02222T } \\
(\text { ATCC 13311) }\end{array}$} & forward & CACGCAGGAAATAACAGGACTT \\
\hline & & reverse & GTGGGCAACCAGCACTAAC \\
\hline \multirow{2}{*}{ Staphylococcus aureus } & \multirow{2}{*}{$\begin{array}{l}\text { NCAIM B.02399 } \\
\text { (ATCC 25923) }\end{array}$} & forward & CAGTATCATCTGTAAATTCACCTC \\
\hline & & reverse & ATCTGTACTCTGTGGAGCTG \\
\hline \multirow{2}{*}{ Listeria monocytogenes } & \multirow{2}{*}{$\begin{array}{l}\text { NCAIM B.01934 } \\
(\text { ATCC 15313) }\end{array}$} & forward & ATGGCACCACCAGCATCTCC \\
\hline & & reverse & TTGTCACTGCATCTCCGTGGTA \\
\hline \multirow{2}{*}{ Zygosaccharomyces bailii } & \multirow{2}{*}{$\begin{array}{c}\text { NCAIM Y.00954T } \\
(\text { ATCC 58445) }\end{array}$} & forward & CAGACATGGTGTTTTGCGCC \\
\hline & & reverse & CGTCCGCCACGAAGTGGTAGA \\
\hline
\end{tabular}

Microbial strains included in the study originated from the National Collection of Agricultural and Industrial Microorganisms (Szent István University, Faculty of Food Science) with the NCAIM reference numbers that corresponds to the ATCC numbers given in brackets. Microbe-specific oligos used to amplify microbes are shown in 5'-3' direction. 


\section{Results and Discussion}

The need for shortening microbe detection and either to supplement or to substitute classical cultivation methods with novel molecular biological approaches in food industry or any other agricultural field is obvious. Due to multiple steps of microbe cultivation, the classical methods may be time-consuming and often yield uncertain results with a consequence of further verification steps [25]. In addition, the sensitivity of most economical molecular methods may be rarely satisfying [3] [17] [30]. We focused our interest on aqueous solution (saline) as inoculation model for drinking water, as the most frequent food product. Applying saline solution prevents spontaneous osmotic stress of microbes preserving their viability longer opposed to water matrix that makes microbes more susceptible to applied lysis treatments [31]. We used two Gram-negative as well as two Gram-positive bacterial strains along with one yeast species as model organisms to demonstrate the potential and efficiency of the chosen methods. At each method the effectiveness of DNA purification was evaluated regarding to the microorganism tested. Furthermore, we compared the earlier published methods and their slightly modified counterparts regarding their lysis efficiency, PCR compatibility, price, simplicity and the time needed for completion. One of the approaches is based on heat-driven lysis of microorganisms and parallel binding of PCR inhibitors using a chelate exchange resin, such as Chelex-100 [4] [6] [8]. In such case, the purity of the DNA is not a main goal, though the qPCR compatibility may be taken into account when analyzing the amplification curves. Even though the Chelex-based methods do not yield pure DNA, and not only proteins, but also other substances might be present in the supernatant, it is important to emphasize that the qPCR inhibitors can be satisfactorily depleted. In certain cases of complex matrices, however, the effectivity of inhibitor depletion has to be experimentally determined as residual organic and some other compounds may substantially impair the polymerase-assisted amplification of desired sequences [32]. Another user-friendly DNA purification method used applies alkaline lysis and ethanol precipitation of the sample DNA [10]. Compared to a commercial kit we managed to ascertain that the used alternative methods may give comparable, or in certain cases, even better Cq values. Under given circumstances, the Gram-negative bacteria were more effectively lysed and amplified using Chelex-100-based methods, which gave better results than the commercial kit. In addition, Chelex-based methods are 10 times more cost-effective and need incomparably less time to carry out the whole procedure from bacterial sample to PCR-ready DNA. In case of Gram-positive bacteria, the Chelex-related approaches were still satisfying, although EtNa-based DNA purifications were a bit more effective in a species-dependent manner, with worse reproducibility. Later on, this could be improved using silica columns as suggested in the original paper of Vingataramin and Frost (2015) instead of pelleting the DNA by centrifugation resulting in a slight but still acceptable elevation of cost per sample [10]. The lysis and amplification of Zygosaccharomyces cells seemed 
to be a bit more efficient using EtNa solution for lysis compared to protocols using Chelate exchange beads. After the comprehensive description we summarize the edification of our results on the used DNA purification techniques according to several interesting aspects.

Results in the case of Gram-negative bacteria: The relatively thin cell wall compared to Gram-positives makes this group of bacteria more sensitive to heat and chemical lysis [31]. According to our results, this characteristic was obviously confirmed because compared to the reference method $\mathrm{Cq}$ values of Gram-negatives were lower than in case of Gram-positives. We experienced better $\mathrm{Cq}$ values after lysis on $100^{\circ} \mathrm{C}$ by chelate-exchange resin-based inhibitor depletion than with any other method including the commercial kit as well. Interestingly, the standard deviation between biological replicates was also significantly lower in this group. The chosen, economically relevant bacteria: Salmonella enterica and Escherichia coli behaved similarly in different protocols. Results are summarized in Table 4 and illustrated in Figure 1.

Results in the case of Gram-positive bacteria: In case of Listeria monocytogenes and Staphylococcus aureus the results of purification processes were not that coherent as seen in case of Gram-negatives. Listeria seemed to be more susceptible to alkaline lysis than to simple heat in contrast to $S$. aureus that showed better Cq values when lysed by heat. Regarding reproducibility this group was more stochastic in our hands, but generally alkaline lysis and ethanol precipitation seemed to be more reliable when using a silica column, which makes the method more expensive. Within this group, the Gram-positive method recommended by the manufacturer of the used commercial kit was proven to be the most effective way of DNA purification, although as seen in Table 4 and Figure 1 , the more economical solutions might also be satisfying in most cases.

Results in case of a yeast species: Although the lysis of yeasts with not exclusively oversimplified methods might be challenging, the Chelex-based approaches and the method presented by Vingataramin and Frost (2015) resulted in excellent Cq values compared to the used commercial kit which is in concordance with observations presented earlier [10]. Regarding the reproducibility of all the methods, elevated standard deviations were observed, except for the GeneJET, which was highly reproducible but lagged behind slightly with the Cq value indicating lower DNA yields. Cq values and standard deviations are summarized in Table 4 and Figure 1.

EtNa vs. EtNa-Chelex. In certain cases the supplementation of DNA dissolving buffer with chelate exchange beads may improve yield or purity of DNA in case of e.g. E. coli, S. aureus, L. monocytogenes or Z. bailii, although the real importance of Chelex-100 in resuspension solution seemed not to be very meaningful, indicating, that the precipitation and the centrifugation steps remove PCR inhibitors sufficiently.

Direct vs. indirect methods. In general, when inoculating colonies into saline or when using culture medium solution directly for DNA purification (unpublished data), there seems to be no need for discarding the solution before 
Table 4. Summary of dCq values of methods applied to different microbes.

\begin{tabular}{|c|c|c|c|c|c|c|c|c|c|c|}
\hline \multirow{3}{*}{$\begin{array}{c}\text { DNA } \\
\text { purification } \\
\text { method }\end{array}$} & \multicolumn{10}{|c|}{ Microbes } \\
\hline & \multicolumn{2}{|c|}{ E. coli } & \multicolumn{2}{|c|}{ S. enterica } & \multicolumn{2}{|c|}{ L. mono } & \multicolumn{2}{|c|}{ S. aureus } & \multicolumn{2}{|c|}{ Z. bailii } \\
\hline & $\begin{array}{c}-\Delta \mathrm{Cq} \\
\text { ave }\end{array}$ & sd & $\begin{array}{c}-\Delta \mathrm{Cq} \\
\text { ave }\end{array}$ & sd & $\begin{array}{c}-\Delta \mathrm{Cq} \\
\text { ave }\end{array}$ & sd & $\begin{array}{l}-\Delta \mathrm{Cq} \\
\text { ave }\end{array}$ & sd & $\begin{array}{c}-\Delta \mathrm{Cq} \\
\text { ave }\end{array}$ & sd \\
\hline GeneJET & - & 0.12 & - & 0.30 & - & 0.29 & - & 0.12 & - & 0.46 \\
\hline Chelex-100 & 0.558 & 0.16 & 0.917 & 0.17 & -2.318 & 0.27 & -1.277 & 0.16 & 1.643 & 0.32 \\
\hline Direct-Chelex & 0.105 & 0.11 & 0.445 & 0.13 & -3.927 & 0.22 & -0.522 & 0.11 & 0.142 & 0.18 \\
\hline EtNa-cf & -1.690 & 0.22 & -1.408 & 0.48 & -2.667 & 0.53 & -1.307 & 0.22 & 3.050 & 1.19 \\
\hline EtNa-cf-Ch. & -1.612 & 0.44 & -1.012 & 0.38 & -2.125 & 0.88 & -0.973 & 0.44 & 0.902 & 0.49 \\
\hline EtNa-cf-column & -0.218 & 0.39 & 0.017 & 0.12 & -0.707 & 0.16 & -1.348 & 0.39 & 0.992 & 0.44 \\
\hline Direct EtNa & -0.493 & 0.11 & -1.093 & 0.65 & -3.625 & 1.13 & -1.458 & 0.11 & 1.248 & 2.11 \\
\hline Direct EtNa-Ch. & -0.378 & 0.12 & -0.925 & 0.36 & -2.990 & 0.90 & -3.992 & 0.12 & 2.403 & 1.59 \\
\hline Direct-EtNa-C. & 0.268 & 0.13 & 0.368 & 0.04 & -0.757 & 0.11 & -0.635 & 0.13 & 1.592 & 0.75 \\
\hline
\end{tabular}

$-\Delta \mathrm{Cq}$ ave means $\mathrm{Cq}$ differences between the average of replicates of the given method and the reference GeneJET kit. Positive values mean better results, namely lower Cq value with the indicated method. In case of $-\Delta \mathrm{Cq}$ ave column, the best $2-3$ is highlighted in grey. Best sd values below 0.3 are also in grey, while worst ones with sd value above 0.6 are framed. Abbreviations: Ch.: Chelex, cf: centrifuge, C.: Column.

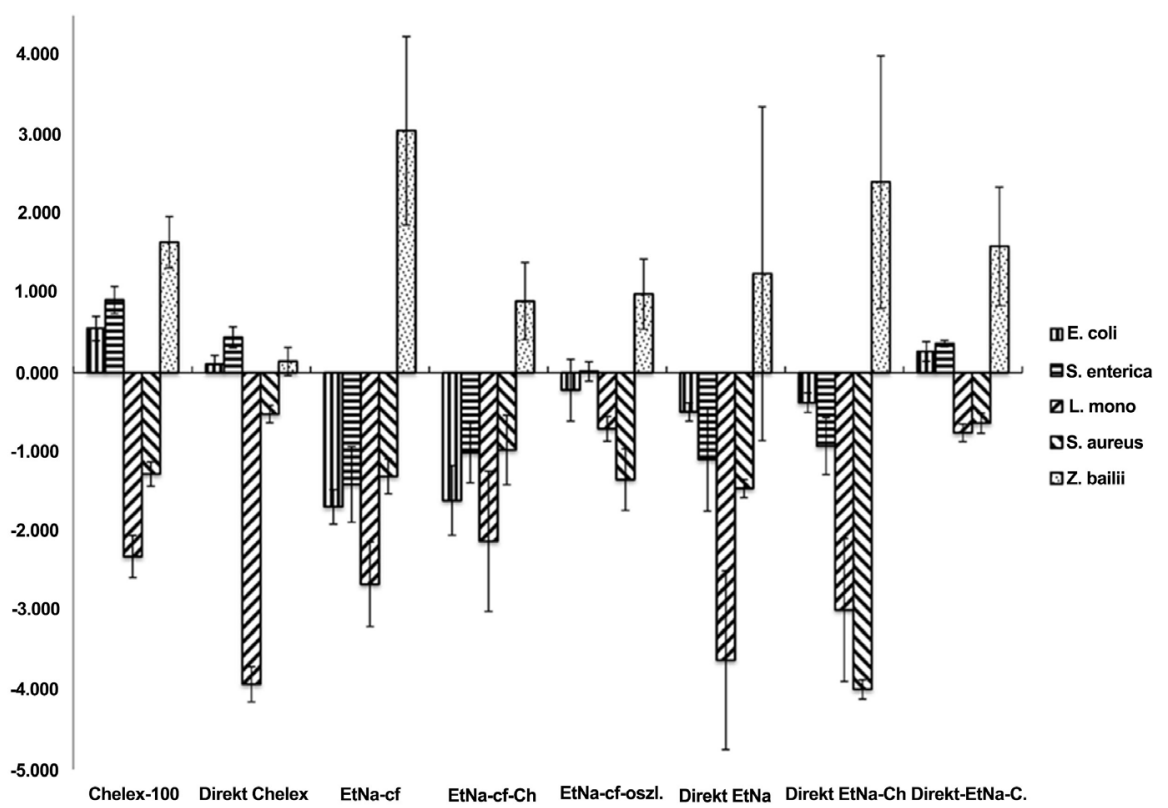

Figure 1. dCq values of methods in case of microbes used in the study.

addition of the lysis buffer, and can be used directly giving the same yield and amplification curve in the subsequent qPCR reactions. The best way of isolating the DNA from cultured microbes is to directly inoculate the lysis solution and go for heating. In this way, the pipetting steps may be reduced to only one (pipetting the lysis solution into empty Eppendorf tube before inoculation) at least in the case of the direct Chelex-100 method. As the EtNa method requires the disposal of the supernatant after DNA precipitation and centrifugation and the 
subsequent pipetting of DNA resuspension solution results in additional two pipetting steps compared to direct Chelex-100.

Time of completion: The fastest method is direct Chelex. According to our experiences for Gram-negative bacteria a two-minutes-long lysis with continuous shaking at $100^{\circ} \mathrm{C}$ is efficient enough (unpublished data) and the time required to get to the qPCR-ready DNA from a colony is less than five minutes (two minutes $100^{\circ} \mathrm{C}$, one minute centrifugation and some handling time). The heat-driven hypo-osmotic lysis of Gram-positive bacteria and yeasts needs to be longer but applying 10 minutes of boiling still allows user to complete the process within a quarter of hour.

Price of DNA isolation out of one sample: EtNa-based methods are undoubtedly the cheapest solutions for DNA isolation regarding only the chemical treatment except for the situation when applying silica column in place of the classical DNA precipitation step with high-speed centrifugation. When considering consumables, e.g. pipette tips and Eppendorf tubes, direct Chelex turns to be preferable from economical point of view (see Table 5). The relative price of GeneJET for one sample was calculated for Gram-negative bacteria. For Grampositive and fungi the prices are higher because of special lysis buffer containing Lysozyme and Zymolase, respectively.

Effectiveness: In certain experiments with Gram-positive bacteria and Zygosaccharomyces EtNa was experienced to be slightly more effective than Chelex-100 or the used commercial kit, although in our hand, the reproducibility of EtNa methods was not really satisfying because the Cq values showed high standard deviation (sd) as compared to other methods, which could be significantly improved by applying silica columns instead of high speed centrifugation similarly to the results of Vingataramin and Frost (2015).

As presented above, beside the Cq value and the reproducibility, the completion time, the number of pipetting steps and the price of a single reaction has also been considered, because molecular biological effectivity is only one side of the coin. The calculated data, regarding previously mentioned parameters, are presented in Table 5. Naturally, the exact numbers in Table 5 may vary individually depending on the source of the materials used including consumables and chemicals, although the relative cost ratios would not change dramatically. According to these results in the case of simple matrixes such as water, PBS, saline solution, culture medium or directly the lysis solution inoculated with the given colony there is no need to pellet the microbes prior to lysis. The direct methods yield equally satisfying results, though depending on the species, some fluctuation in Cq values might occur. No doubt, the Chelex-100 based methods were outstanding from the economical (cost, time and handling) point of view.

\section{Conclusion}

Taken together, several cheap, short and excellent recipes are available for microbial DNA isolation from physiological salt solution, colonies or bacterial culture media. Besides the well-formulated commercial kits, the PCR-ready DNA 
Table 5. Comparison of methods used in the study.

\begin{tabular}{cccc}
\hline DNA purification method & \#Pipetting steps & Time required (min) & $\begin{array}{c}\text { Relative estimated Price } \\
\text { to 1 "sample" }\end{array}$ \\
\hline GeneJET & $>12$ & $60-180$ & 11.94 \\
Chelex-100 & 3 & 22 & 1.3 \\
Direct-Chelex & 2 & 15 & 1 \\
EtNa-cf & 5 & 37 & 1.63 \\
EtNa-cf-Ch. & 5 & 37 & 1.94 \\
EtNa-cf-column & 5 & 30 & 3.13 \\
Direct EtNa & 4 & 30 & 1.33 \\
Direct EtNa-Ch. & 4 & 30 & 1.64 \\
Direct EtNa-C. & 4 & 23 & 2.83 \\
\hline
\end{tabular}

The relative price of GeneJET for one sample was calculated for Gram-negative bacteria. In the case of Gram-positives and fungi the prices are higher because of special lysis buffer containing Lysozyme and Zymolase, respectively. As currency and other circumstances have an effect on the prices of the components, the cheapest method, Direct-Chelex was regarded as 1 unit and others are shown in proportion accordingly. The Pipetting steps column include the handling of sample as the first pipetting. Abbreviations: Ch.: Chelex, cf: centrifuge, C.: Column.

can be easily purified with these alternative methods within much less than 20 minutes. While avoiding compromises concerning the DNA yield when using the above mentioned approaches, both the prize and duration time of DNA preparation can be minimized. Last but not least-due to lower consumption of plastic material as well as harmful solutions-the carbon footprint of these procedures is significantly lower. It is difficult to claim one of the above described experimentally analyzed methods for the best approach at any situation but for general purposes using simple matrixes, the direct Chelex method is undoubtedly a good option to obtain purified DNA for PCR and/or qPCR amplification. When the starting material is more complex, additional matrix-dependent treatments may be required prior to DNA purification. Nevertheless, the simple methods mentioned here are good starting points for the development of matrix-specific or more general cost-effective DNA purification procedures aiming the detection of pathogenic or indicative microbes from any sample. In addition to these considerations, the simple methods consisting of only several pipetting steps have a good potential for adopting automatic procedures and robotic handling providing high throughput sample processing if required [33].

\section{Conflicts of Interest}

No conflict of interest declared.

\section{References}

[1] Rohde, A., Hammerl, J.A., Appel, B., Dieckmann, R. and Al Dahouk, S. (2015) Fishing for Bacteria in Food-A Promising Tool for the Reliable Detection of $\mathrm{Pa}$ thogenic Bacteria? Food Microbiology, 46, 395-407. 
https://doi.org/10.1016/j.fm.2014.09.002

[2] Franco-Duarte, R., et al. (2019) Advances in Chemical and Biological Methods to Identify Microorganisms-From Past to Present. Microorganisms, 7, 130.

https://doi.org/10.3390/microorganisms7050130

[3] Law, J.W.-F., Ab Mutalib, N.-S., Chan, K.-G. and Lee, L.-H. (2015) Rapid Methods for the Detection of Foodborne Bacterial Pathogens: Principles, Applications, Advantages and Limitations. Frontiers in Microbiology, 5, 770.

https://doi.org/10.3389/fmicb.2014.00770

[4] Walsh, P.S., Metzger, D.A. and Higuchi, R. (1991) Chelex 100 as a Medium for Simple Extraction of DNA for PCR-Based Typing from Forensic Material. Biotechniques, 10, 506-513.

[5] Phillips, K., McCallum, N. and Welch, L. (2012) A Comparison of Methods for Forensic DNA Extraction: Chelex-100(R) and the QIAGEN DNA Investigator Kit (Manual and Automated). Forensic Science International: Genetics, 6, 282-285. https://doi.org/10.1016/j.fsigen.2011.04.018

[6] Musapa, M., et al. (2013) A Simple Chelex Protocol for DNA Extraction from Anopheles spp. Journal of Visualized Experiments, No. 71, e3281.

https://doi.org/10.3791/3281

[7] Miller, D.N., Bryant, J.E., Madsen, E.L. and Ghiorse, W.C. (1999) Evaluation and Optimization of DNA Extraction and Purification Procedures for Soil and Sediment Samples. Applied and Environmental Microbiology, 65, 4715-4724. https://doi.org/10.1128/AEM.65.11.4715-4724.1999

[8] Lamballerie, X., Zandotti, C., Vignoli, C., Bollet, C. and de Micco, P. (1992) A One-Step Microbial DNA Extraction Method Using “Chelex 100” Suitable for Gene Amplification. Research in Microbiology, 143, 785-790. https://doi.org/10.1016/0923-2508(92)90107-Y

[9] Coombs, N.J., Gough, A.C. and Primrose, J.N. (1999) Optimisation of DNA and RNA Extraction from Archival Formalin-Fixed Tissue. Nucleic Acids Research, 27, e12.

[10] Vingataramin, L. and Frost, E.H. (2015) A Single Protocol for Extraction of gDNA from Bacteria and Yeast. BioTechniques, 58, 120-125. https://doi.org/10.2144/000114263

[11] Engvall, E. and Perlmann, P. (1972) Enzyme-Linked Immunosorbent Assay, Elisa: III. Quantitation of Specific Antibodies by Enzyme-Labeled Anti-Immunoglobulin in Antigen-Coated Tubes. The Journal of Immunology, 109, 129-135.

https://doi.org/10.1016/B978-0-08-016876-0.50102-X

[12] Pavlovic, M., Huber, I., Konrad, R. and Busch, U. (2013) Application of MALDI-TOF MS for the Identification of Food Borne Bacteria. The Open Microbiology Journal, 7, 135-141. https://doi.org/10.2174/1874285801307010135

[13] Singhal, N., Kumar, M., Kanaujia, P.K. and Virdi, J.S. (2015) MALDI-TOF Mass Spectrometry: An Emerging Technology for Microbial Identification and Diagnosis. Frontiers in Microbiology, 6, 791. https://doi.org/10.3389/fmicb.2015.00791

[14] Wenning, M., Breitenwieser, F., Konrad, R., Huber, I., Busch, U. and Scherer, S. (2014) Identification and Differentiation of Food-Related Bacteria: A Comparison of FTIR Spectroscopy and MALDI-TOF Mass Spectrometry. Journal of Microbiological Methods, 103, 44-52. https://doi.org/10.1016/j.mimet.2014.05.011

[15] Mechaly, A., Cohen, H., Cohen, O. and Mazor, O. (2016) A Biolayer Interferometry-Based Assay for Rapid and Highly Sensitive Detection of Biowarfare Agents. 
Analytical Biochemistry, 506, 22-27. https://doi.org/10.1016/j.ab.2016.04.018

[16] Dua, P., et al. (2016) Cell-SELEX Based Identification of an RNA Aptamer for Escherichia coli and Its Use in Various Detection Formats. Molecules and Cells, 39, 807-813. https://doi.org/10.14348/molcells.2016.0167

[17] Banerjee, P., Sulaiman, I.M., Schneider, G., Ray, U. and Jagadeesan, B. (2017) Novel Microbial Diagnostic Methods for Clinical, Environmental, and Food Samples. BioMed Research International, 2017, Article ID: 3942801. https://www.hindawi.com/journals/bmri/2017/3942801/abs/ https://doi.org/10.1155/2017/3942801

[18] Rajapaksha, P., Elbourne, A., Gangadoo, S., Brown, R., Cozzolino, D. and Chapman, J. (2019) A Review of Methods for the Detection of Pathogenic Microorganisms. Analyst, 144, 396-411. https://doi.org/10.1039/C8AN01488D

[19] Farber, J.M. and Peterkin, P.I. (1991) Listeria Monocytogenes, a Food-Borne Pathogen. Microbiological Reviews, 55, 476-511.

[20] Archer, G.L. (1998) Staphylococcus aureus. A Well-Armed Pathogen. Clinical Infectious Diseases, 26, 1179-1181. https://doi.org/10.1086/520289

[21] Humphrey, T. (2004) Salmonella, Stress Responses and Food Safety. Nature Reviews Microbiology, 2, 504-509. https://doi.org/10.1038/nrmicro907

[22] Kaper, J.B., Nataro, J.P. and Mobley, H.L. (2004) Pathogenic Escherichia coli. Nature Reviews Microbiology, 2, 123-140. https://doi.org/10.1038/nrmicro818

[23] Fleet, G.H. (2007) Yeasts in Foods and Beverages: Impact on Product Quality and Safety. Current Opinion in Biotechnology, 18, 170-175. https://doi.org/10.1016/j.copbio.2007.01.010

[24] Hoffmann, S., Batz, M.B. and Morris, J.G. (2012) Annual Cost of Illness and Quality-Adjusted Life Year Losses in the United States Due to 14 Foodborne Pathogens. Journal of Food Protection, 75, 1292-1302. https://doi.org/10.4315/0362-028X.JFP-11-417

[25] Sanders, E.R. (2012) Aseptic Laboratory Techniques: Plating Methods. Journal of Visualized Experiments, No. 63, e3064. https://doi.org/10.3791/3064

[26] Miras, I., Hermant, D., Arricau, N. and Popoff, M.Y. (1995) Nucleotide Sequence of iagA and iagB Genes Involved in Invasion of HeLa Cells by Salmonella enterica subsp. enterica ser. Typhi. Research in Microbiology, 146, 17-20.

https://doi.org/10.1016/0923-2508(96)80267-1

[27] Rawsthorne, H. and Phister, T.G. (2006) A Real-Time PCR Assay for the Enumeration and Detection of Zygosaccharomyces bailii from Wine and Fruit Juices. International Journal of Food Microbiology, 112, 1-7. https://doi.org/10.1016/j.ijfoodmicro.2006.05.003

[28] Sandhya, S., Chen, W. and Mulchandani, A. (2008) Molecular Beacons: A Real-Time Polymerase Chain Reaction Assay for Detecting Escherichia coli from Fresh Produce and Water. Analytica Chimica Acta, 614, 208-212.

https://doi.org/10.1016/j.aca.2008.03.026

[29] Quero, G.M., Santovito, E., Visconti, A. and Fusco, V. (2014) Quantitative Detection of Listeria Monocytogenes in Raw Milk and Soft Cheeses: Culture-Independent versus Liquid- and Solid-Based Culture-Dependent Real Time PCR Approaches. LWT-Food Science and Technology, 58, 11-20. https://doi.org/10.1016/j.lwt.2014.03.005

[30] Elizaquivel, P., Aznar, R. and Sanchez, G. (2014) Recent Developments in the Use of Viability Dyes and Quantitative PCR in the Food Microbiology Field. Journal of 
Applied Microbiology, 116, 1-13. https://doi.org/10.1111/jam.12365

[31] Wood, J.M. (2015) Bacterial Responses to Osmotic Challenges. Journal of General Physiology, 145, 381-388. https://doi.org/10.1085/jgp.201411296

[32] Hu, Q., Liu, Y., Yi, S. and Huang, D. (2015) A Comparison of Four Methods for PCR Inhibitor Removal. Forensic Science International: Genetics, 16, 94-97. https://doi.org/10.1016/j.fsigen.2014.12.001

[33] Wallinger, C., et al. (2017) Evaluation of an Automated Protocol for Efficient and Reliable DNA Extraction of Dietary Samples. Ecology and Evolution, 7, 6382-6389. https://doi.org/10.1002/ece3.3197 